\title{
Anabases
}

ANABASES Traditions et réceptions de l'Antiquité

29 | 2019

Varia

\section{Entre Clio et Thémis : entretien avec Dario \\ Mantovani (Collège de France)}

Hélène Ménard

\section{(2) OpenEdition}

1 Journals

Édition électronique

URL : https://journals.openedition.org/anabases/9329

DOI : 10.4000/anabases.9329

ISSN : 2256-9421

Éditeur

E.R.A.S.M.E.

Édition imprimée

Date de publication : 14 avril 2019

Pagination : 365-368

ISSN : 1774-4296

\section{Référence électronique}

Hélène Ménard, «Entre Clio et Thémis : entretien avec Dario Mantovani (Collège de France) »,

Anabases [En ligne], 29 | 2019, mis en ligne le 14 avril 2021, consulté le 06 novembre 2021. URL

http://journals.openedition.org/anabases/9329; DOI : https://doi.org/10.4000/anabases.9329

(C) Anabases 

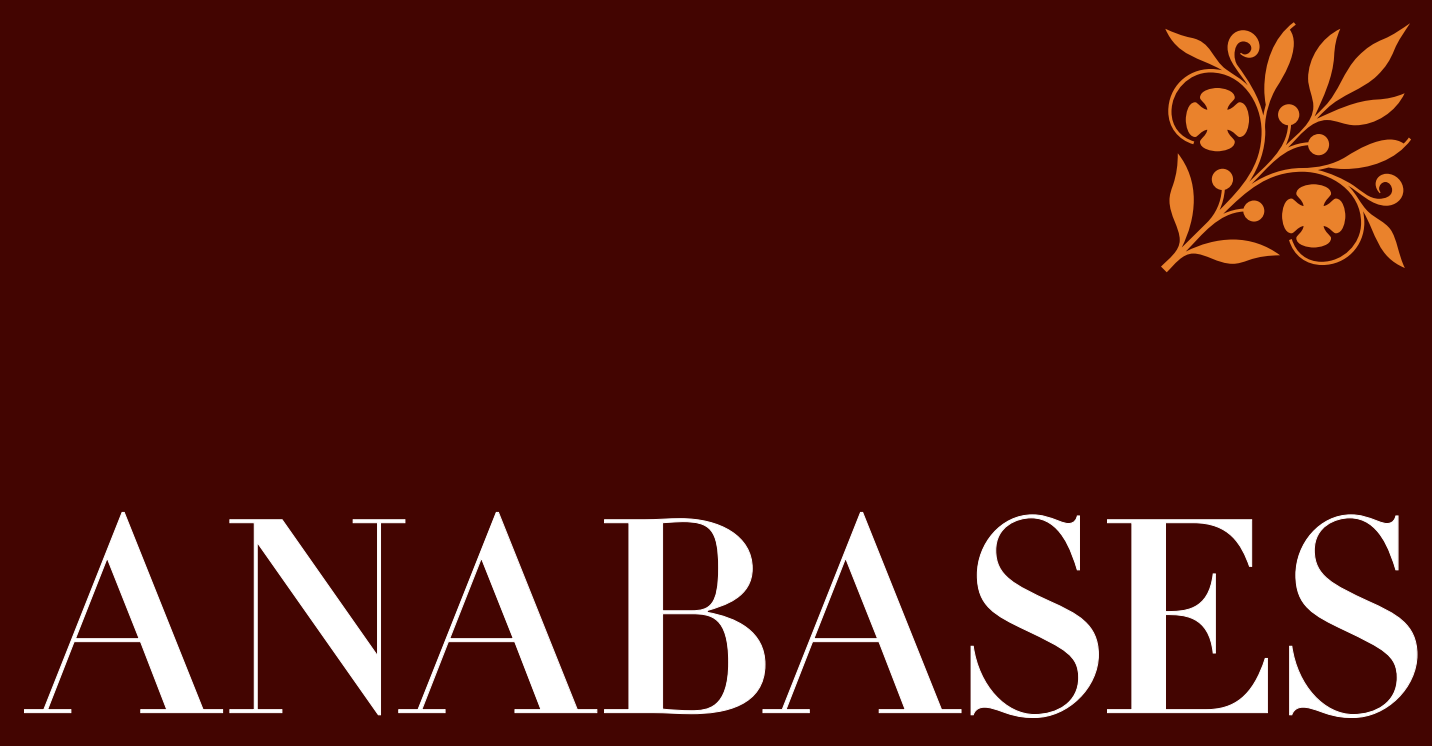

Traditions et Réceptions de l'Antiquité

\section{No29 \\ 2019}

Juliette Ernst Sculptures onctueuses de Meekyoung Shin Théâtre antique et travaux savants dans la Première modernité L'Antiquité dans la peinture (17911880) Réception d'Ovide Pierre Grimal 
ANABASES

Traditions et Réceptions de l'Antiquité

Revue de l'équipe de recherche E.R.A.S.M.E.

Université Toulouse-Jean Jaurès (UT2J)

Anabases dispose d'un Comité de lecture international. Chaque article envoyé à la rédaction est soumis, une fois anonymisé, à l'expertise de deux spécialistes qui rendent un rapport écrit. Les deux rapports anonymisés sont transmis à l'auteur qui tient compte des observations en vue de la publication.

\section{Comité SCIEnTIFIQUe}

Germaine Aujac (université Toulouse-Jean Jaurès : histoire de la géographie et des sciences antiques)

Florence Bouchet (université Toulouse-Jean Jaurès : littérature médiévale)

Hinnerk BruHns (CNRS : histoire économique et sociale ancienne et contemporaine)

Paulo Butti de Lima (université de Bari : historiographie et réception de l'Antiquité)

Luciano CANFora (université de Bari : littérature et histoire anciennes, historiographie)

Giovanna Ceserani (Stanford University : histoire intellectuelle et historiographie de la tradition classique)

Temístocles Cezar (université de Porto Alegre : historiographie moderne)

Serafina Сиомо (University of London, Birkbeck College : histoire des mathématiques et des sciences)

Paul Demont (université de Paris Sorbonne : philologie grecque et héritage classique)

Marie-Laurence Desclos (université de Grenoble II : philosophie de l'Antiquité)

Olivier Devillers (université de Bordeaux 3 - Michel-de-Montaigne : littérature et historiographie latines)

Andrea Giardina (Istituto italiano di scienze umane : histoire du monde romain et de ses réceptions)

Ève Gran-Aymerich (aibl : histoire de l'archéologie et des transferts culturels)

François HaRTog (EHEss : historiographie ancienne et moderne)

Geneviève Hoffmann (université de Picardie : histoire des mondes grecs)

Christian JACOB (CNRS/EHEss : histoire comparée et épistémologie des savoirs)

Suzanne Marchand (Louisiana State University : histoire du classicisme et de l'orientalisme)

Wilfried Nippel (Humboldt Universität Berlin : histoire et historiographie de l'Antiquité)

Sylvie Pittia (université de Paris I-Panthéon Sorbonne : histoire et historiographie du monde romain)

Stéphane Ratri (université de Franche-Comté - Besançon : philologie et héritage latin)

Comité de RÉdaction

Jacques Alexandropoulos, Marielle de Béchillon, Corinne Bonnet, Laurent Bricault, Clément Bur,

Philippe Foro, Adeline Grand-Clément, Anne-Hélène Klinger-Dollé, Véronique Krings,

Thibaud Lanfranchi, Claudine Leduc, Pascal Payen, Grégory Reimond, Catherine Valenti

Éditeur RESPonsable

Pascal PAYen

Université Toulouse-Jean Jaurès (UT2J)

SECRÉTARIAT DE RÉDACTION

Anthony Andurand / Clément Bertau-Courbières / Corinne Bonnet / Clément Bur /

Adeline Grand-Clément / Anne-Hélène Kuinger-Dollé / Véronique Krings /

Catherine Valenti (université Toulouse-Jean Jaurès) / Noémie VillacÈQue (université de Reims)

Sites Web

http://plh.univ-tlse2.fr

Revues.org : http://anabases.revues.org

Aвonnement et vente aU numéro

Éditions De Boccard - 4, rue de Lanneau - 75005 Paris

info@deboccard.com - www.deboccard.com

Tél. : 0033/(0)143260037 - Fax : 0033/(0)143548583 




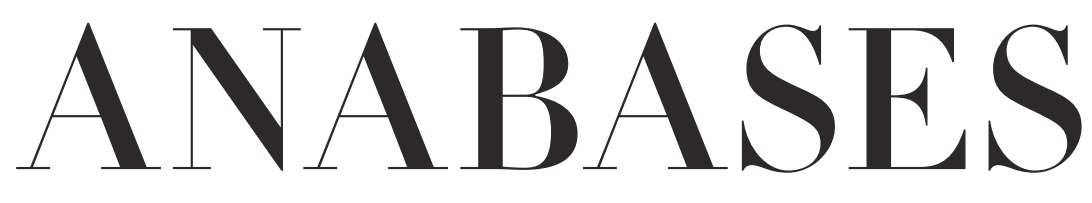

Traditions et Réceptions de l'Antiquité

$$
\begin{aligned}
& N \circ 29 \\
& 2019
\end{aligned}
$$

\section{E.R.A.S.M.E.}

Université Toulouse - Jean Jaurès 



\section{Sommaire}

$\mathrm{N}^{\circ} 29-2019$

\section{Historiographie et identités culturelles}

Ilse Hilbold

Les archives d'une bibliographe des sciences de l'Antiquité :

Juliette Ernst et la fabrique des relations internationales . . . . . . . . . I I3

Vivien LONGHI

La crise, une notion politique héritée des Grecs ? . . . . . . . . . . . 2I

Mireille Lacave-Allemand et Michel Lacave,

L’Antiquité dans la peinture en France, I79I-I880 :

une analyse quantitative à travers les Salons et les Prix de Rome . . . . . 37

Tiphaine Besnard

Du Weathering Project aux autoportraits en Venus :

Les sculptures onctueuses et savonneuses de Meekyoung Shin . . . . . 7 I

\section{Traditions du patrimoine antique}

Dossier dirigé par Pascale Paré-Rey et Malika Bastin-Hammou,

“La réception du théâtre antique dans les travaux savants de l’Europe

de la Première modernité »

Malika Bastin-Hammou et Pascale Paré-Rey

“ La réception du théâtre antique dans les travaux savants

de l'Europe de la Première modernité » . . . . . . . . . . . . . . . . 89

Kevin Bovier

Rétablir la métrique de Térence au $\mathrm{XvI}^{\mathrm{e}}$ siècle :

le cas du Iudicium de Glaréan (1540) . . . . . . . . . . . . . . . . . . . . . . 93 
Brice Denoyer

L'héritage de la métrique antique

dans l'alexandrin français au xvie siècle . . . . . . . . . . . . . IO7

Giovanna Di Martino

Vittorio Alfieri's tormented relationship with Aeschylus:

Agamennone between Tradition and Innovation . . . . . . . . . . . . . . . I2I

Marco Duranti

La condanna del prologo diegetico euripideo dagli scoli antichi

ai trattati del Cinquecento . . . . . . . . . . . . . . .

Rosario López Gregoris

L'influence de l' Arte nuevo de hacer comedias de Lope de Vega

dans l'usage des modèles classiques latins en Espagne

pendant le Siècle d'or et le Baroque » . . . . . . . . . . . . . . . . I49

Cressida Ryan

Sophoclean scholarship as a tool

to interpret eighteenth-century England . . . . . . . . . . . . . . г 6 I

Záviš ŠumaN

Axiologie critique de La Mesnardière . . . . . . . . . . . . . . . . . . . . I79

\section{Archéologie des savoirs}

Dossier dirigé par Cristina Noacco

“2000 ans déjà... Aspects de la réception d'Ovide » . . . . . . . . . . . I93

\section{La réception d'Ovide au Moyen Âge}

Jean-Marie Fritz et Cristina NoAcco

Lire Ovide au xiI ${ }^{\mathrm{e}}$ siècle : Arnoul d'Orléans

commentateur des Métamorphoses . . . . . . . . . . . . . . . $\quad{ }_{195}$

Franck Coulson

Le mythe de Pythagore dans le commentaire

“Vulgate » des Métamorphoses . . . . . . . . . . . . . . . . . . . . . . 2I

Marylène Possamaï

Comment éditer l'Ovide moralisé :

le problème de la mise en page du manuscrit Rouen Bm O.4 . . . . . . . 225

Anneliese Pollock Renck

Les Hérö̈des à la fin du Moyen Âge : pour une définition élargie de l'acte traducteur . . . . . . . . . . . . . . . . . . 239 
II. La réception d'Ovide à l'époque moderne

Fátima Díez Platas et Patricia Meilán Jácome

Le poète dans son œuvre. Ovide dans les images des Fasti

et des Tristia entre les Xv et $\mathrm{xvI}^{\mathrm{e}}$ siècles . . . . . . . . . . . . . . . . . 255

Ana Paula Rebelo Correia

Les représentations des Métamorphoses d'Ovide

dans les azulejos portugais. Influence des modèles gravés français . . . . 269

Sarah ReY

Figures d'Orphée au cinéma . . . . . . . . . . . . . . . . . . . 277

\section{Actualités et débats}

Marine LE BAIL

La modernité littéraire serait-elle affaire d'Antiquité(s) ?

Euvres \& Critiques: La contribution de l'archéologie à la genèse

de la littérature moderne, XLII, I, René Sternke dir., 20I7, 338 p. . . . . . . 2 29I

\section{Lire, relire la bibliothèque des sciences de l'Antiquité}

Éric Morvillez

“Les Horti Tauriani de Pierre Grimal

ou les prémices des Jardins romains » . . . . . . . . . . . . . . . . 30I

Pierre Grimal

“Les Horti Tauriani. Étude topographique sur la région

de la Porte Majeur ",MEFRA, tome 53, rg36. p. 25o-286 . . . . . . . . . . . 3 3i3

\section{L'atelier de l'histoire : chantiers historiographiques}

L'Antiquité au musée (coordonné par Adeline Grand-Clément) (6)

Aurélie Rodes, Catherine Valenti

Les Gaulois au musée . . . . . . . . . . . . . . . . . . . .

355

L'Atelier des doctorants (coordonné par Adeline Grand-Clément) (16)

Andrea Avalli

La question étrusque dans l'Italie fasciste $\ldots \ldots \ldots$. . . . . . . 360 
Droit et réception de l'Antiquité

(coordonné par Marielle de Béchillon et Hélène Ménard) (6)

Entre Clio et Thémis. Entretien avec Dario Mantovani, réalisé par

Hélène Ménard (Maître de Conférences d'Histoire romaine, à l'Université

Paul Valéry - Montpellier III), le 22 juin 20I8, à l'occasion de la parution

aux Belles Lettres du livre Les juristes écrivains de la Rome antique.

Les ouvres des juristes comme littérature (juin 20ı8) et de la création

de la chaire “ Droit, culture et société de la Rome antique »

au Collège de France $\left(\mathrm{I}^{\mathrm{er}}\right.$ novembre $\left.20 \mathrm{I} 8\right) \ldots$. . . . . . . . . . . . .

\section{Comptes rendus}

Philippe Borgeaud et Sara Petrella

Le singe de l'autre.

Du sauvage américain à l'histoire comparée des religions (A. Guedon) . . $\quad 37 \mathrm{I}$

Roberta Casagrande-Kim, Samuel Thrope et Raquel Ukeles (éd.)

Romance and reason. Islamic transformations of the classical past

(Cl. Bertau-Courbières) . . . . . . . . . . . . . . . . . . . . .

Hinnerk BruHns

Max Webers historische Sozialökonomie.

L'économie de Max Weber entre histoire et sociologie (Th. Lanfranchi) . . 374

Andrea Cozzo

Riso e sorriso, e altre saggi sulla nonviolenza nella Grecia antica,

(Fr. Pr. Barone) . . . . . . . . . . . . . . . . . . . 377

Franz Cumont

Manichéisme (St. Ratti) . . . . . . . . . . . . . . . 378

Emmanuelle HÉnin et Valérie NAAs (dir.)

Le mythe de l'art antique (Cl. Evrard) . . . . . . . . . . . . . . . . . 380

Jacques Jouanna, Henri Lavagne, Alain Pasquier,

Véronique SchiLtz et Michel Zink (éd.)

Au-delà du Savoir : Les Reinach et le Monde des Arts (G. Hoffmann) . . . .

382

Mario Liverani

Imagining Babylon: The Modern Story of an Ancient City (C.Bonnet) . . . 386

Françoise-Hélène Massa-Pairault, Claude Pouzadoux (Dir.)

Géants et Gigantomachie entre Orient et Occident (C.Giovénal) . . . . . . .

Scott McGill, Joseph Pucci (éd.)

Classics renewed. Reception and Innovation in the Latin Poetry

ofLate Antiquity (S. Clément-Tarantino) . . . . . . . . . . . . . . . . . . . 
Maxwell T. PAule

Canidia, Rome's First Witch (C. Landrea) . . . . . . . . . . . . . . . . . . . 39г

Jessica Priestley, Vasiliki Zali (éd.)

Brill's Companion to the Reception of Herodotus in Antiquity

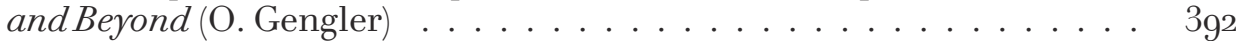

Salvatore QuAsimodo

La Lyre grecque $(\mathrm{M}$. Bianco) . . . . . . . . . . . . . . . . . . 395

Brett M. Rogers, Benjamin Eldon Stevens (éd.)

Classical Traditions in Modern Fantasy (M. Scapin) . . . . . . . . . . . . . 397

Maria Teresa Schettino et Céline UrLacher-Becht (dir.)

Ipse dixit. L'autorité intellectuelle des Anciens : affirmation,

appropriations, détournements (C. Psilakis) . . . . . . . . . . . . . 398

Guy G. Stroumsa

Religions d'Abraham : histoires croisées (D. Lorin) . . . . . . . . . . . . 400

Jean Yvonneau (éd.)

La Muse au long couteau. Critias, de la création littéraire

au terrorisme d'État (G. Hoffmann) . . . . . . . . . . . . . . 405

Résumés . . . . . . . . . . . . . . . . . . . . . 409

Index .............................. 423 



\section{L'atelier de l'histoire : \\ chantiers historiographiques}



à la Résistance avec le parti communiste et, dans les années I95o, il continue sa critique du mythe étrusque en défendant le réalisme socialiste. Une confrontation des parcours de Pallottino et Bianchi Bandinelli permettra ainsi d'étudier les changements et les continuités des représentations de l'histoire ancienne dans la culture de l'Italie postfasciste, en lien avec une société qui est en train de changer.

\section{Andrea Avalli}

Doctorant de l'Université de Gênes

(DAFIST) et de l'Université de Picardie-Jules Verne (TrAme)

Via Bruzzone 21/18

17100 Savona $(\mathrm{SV})$ - Italie

xba@hotmail.it

\section{Droit et réception de l'antiquité}

\section{Entre Clio et Thémis : entretien avec Dario Mantovani (Collège de France)}

Cet entretien a été réalisé le 22 juin 2018 avec Dario Mantovani, juriste historien, par Hélène Ménard, Maître de conférences d'Histoire romaine à l'Université Montpellier 3 Paul Valéry à l'occasion de la parution aux Belles Lettres du livre Les juristes écrivains de la Rome antique. Les ouvres des juristes comme littérature, et de la création de la chaire “ Droit, culture et société de la Rome antique » au Collège de France.

- Étudier le droit romain au xx siècle apparaît à la fois comme une évidence (car il constitue le substrat dans lequel s'enracinent la plupart des droits d'Europe occidentale en vigueur entre le XI 'eiècle et le XIX siècle) et comme une difficulté : il s'agit d'un mode de pensée et d'une langue propres, qui apparaissent souvent comme peu accessibles voire hermétiques, notamment aux étudiants et même aux chercheurs d'autres disciplines. Quelles sont d'après vous les éléments du droit romain qui rendent son approche parfois difficile, mais aussi intellectuellement stimulante?

D'abord, le droit romain est incorporé dans une masse de textes assez imposante, quoique réduite par rapport à ce qui circulait. Ensuite, et surtout, le système juridique romain ne fonctionnait pas de façon déductive, à partir de normes. Il y avait, bien entendu, des lois et de règles, mais, pour la plupart, les solutions étaient données par rapport à des 
situations assez précises et spécifiques, des cas d'espèces : un particulier consultait un juriste, lui aussi un particulier, pour obtenir la solution à son problème. Pour un lecteur moderne, lire le problème posé, puis tout de suite la réponse du juriste romain, est un peu étonnant, comme s'il y avait une sorte de lacune. On est amené à se demander : d'où le juriste puisait-t-il sa réponse, la solution du problème qu'on lui avait posé ? C'est là toute la difficulté. Car c'est seulement par une lecture très étendue des textes des juristes romains qu'on commence à entrer dans leur mentalité et à se rendre compte que les réponses qu'ils donnaient à des cas très précis découlaient d'un raisonnement. Ce raisonnement était rationnel et vérifiable, construit sur deux piliers : le premier, ce sont les schémas d'argumentation, par ex., la réduction à l'absurde, l'analogie ou la définition par rapport à l'étymologie, donc des moyens qui sont communs à toutes sortes de raisonnements et que, dans l'Antiquité, les jeunes gens apprenaient dans les cours de rhétorique. Ce qui représentait la spécificité du discours juridique, c'étaient en revanche les valeurs à partir desquelles les juristes appliquaient ces schémas argumentatifs. Souvent, lorsque l'on parle du raisonnement des juristes romains (mais également lorsque l'on analyse les arrêts des juges contemporains) on a tendance à se focaliser sur les schémas, mais ils sont vides pour ainsi dire : il faut donc y incorporer les valeurs. Et les valeurs des juristes sont un peu plus difficiles à saisir. Je n'en cite qu'une, très répandue, c'est-à-dire que "personne ne doit s'enrichir en causant un dommage à autrui ». Ce principe, apparemment vague, est un dispositif propre à créer nombre de solutions. Le discours des juristes romains peut donc paraître difficile, mais son importance tient à ce type de raisonnement qui incorpore à la fois des outils d'argumentation et des valeurs. Et le réactiver nous met en contact avec un des discours les plus développés que l'humanité a jamais élaborés sur la justice, prise dans sa fonction très concrète de résolution des conflits entre les individus. Et comme il arrive souvent, cet acte de réactiver “ des pensées déjà bien pensées »-sans que cela implique de les mener au même but - nous permet de mûrir notre propre capacité à élaborer notre propre pensée.

- Votre ouvrage sur les juristes écrivains s'attache à retrouver la pensée juridique de ces auteurs dont seuls des fragments des œuvres nous sont parvenus, sauf cas exceptionnel comme celui du manuel de Gaius. Cette pensée juridique, vous l'abordez à travers la façon qu'elle a d'être écrite, à travers son écriture. D'où vient cet intérêt pour une approche littéraire d'oeuvres souvent simplement abordées sous l'angle de leur technicité?

On peut distinguer deux aspects : ce qui a à faire avec mon expérience et ma formation, et ce qui a à faire avec l'objet lui-même. Au lycée, en Italie,j'ai étudié la littérature grecque et latine, la littérature italienne et européenne, doncj'ai reçu une éducation qui est en principe sensible au fait littéraire. Cela m'a rendu conscient de ce que la pensée est influencée par sa forme littéraire. Quant à la nature de l'objet étudié, il faut tenir compte de ce que le droit romain a été étudié (depuis le $\mathrm{xI}^{\mathrm{e}}$ siècle, dans les universités italiennes et européennes) par des juristes. Les juristes ont toujours le désir de saisir le noyau normatif des textes romains et de le désincarner de sa forme littéraire, un peu comme si il s'agissait de réduire le discours des juristes le plus possible à la forme de normes juridiques. Mais les juristes romains n'étaient pas des législateurs et donc leur discours était plus ou moins influencé par un souci littéraire. Littéraire ne signifie pas souci d'élégance ou de style agréable. C'est un effort de clarté et d'efficacité. Les livres des juristes, bien qu'appartenant à un genre 
technique, ne renonçaient pas à appliquer les règles rhétoriques à un certain degré. Ce sont les présupposés qui m’ont convaincu qu'il fallait appliquer aux juristes romains les mêmes interrogations et les mêmes outils dont profitent les latinistes pour interroger les textes littéraires. J'ose dire que les textes des juristes sont encore plus stimulants lus sous cet angle, parce que - étant justement des textes techniques - tout ce qui s'écarte de la neutralité de l'expression est très significatif. Dans ce goût pour le dévoilement de la pensée par le biais de l'expression, on peut même aller plus loin, lorsque l'on se penche non pas sur les textes juridiques eux-mêmes, mais sur les gloses qui leur étaient parfois appliquées. Elles donnent un grand plaisir à celui qui s'essaie à les déchiffrer, parce que l'effort de concentrer sa pensée dans ce type d'annotations marginales fait qu'elles sont énigmatiques (elles étaient souvent censées même n'être pas lues par d'autres lecteurs que celui qui les avait rédigées comme aide-mémoire). Donc le style des gloses stimule beaucoup mon goût pour la restitution, pour retrouver le sens que l'auteur antique si loin de nous avait voulu donner à ce qu'il écrivait. Bref. Les textes techniques, dont les textes juridiques font partie, nous lancent un défi d'interprétation qu'il faut accepter, et la façon meilleure de s'en sortir est de se munir des mêmes outils que ceux que les latinistes ont peaufinés en lisant les textes littéraires.

- L'un des apports originaux de votre ouvrage est de redécouvrir les ouvrages des juristes comme des livres circulant, sous un format précis qui les rendait reconnaissables, et donc avec une mise en forme spécifique. Rendre leur matérialité aux livres juridiques a-t-il constitué une étape importante dans votre réflexion et pourquoi?

Les historiens du droit s'appuient normalement sur les éditions modernes des textes juridiques romains, d'abord le Digeste de Justinien, puis les autres parties du Corpus Iuris Civilis et les autres sources dont nous disposons. C'est bien compréhensible, mais c'est une autre façon de s'éloigner du côté littéraire, de la spécificité des sources juridiques. Il faut les remettre dans le cadre originel, et pour faire cela il faut partir de cette question : qui étaient les lecteurs préconisés par les juristes, pour qui écrivaient-ils? Le public préconisé, évidemment, avait des conséquences sur la façon dont ils écrivaient, et cette question a à faire aussi avec le côté matériel des livres. Il faut se demander sur quels supports, de quelle façon, avec quelle mise en page ces livres juridiques circulaient. Avoir eu la possibilité de travailler avec des papyrologues et de m'approcher ainsi des documents, en revenant du texte imprimé au texte tel qu'il avait été, à un moment donné, dans les mains des lecteurs anciens, a contribué à me convaincre qu'il faut abandonner la perspective “ moderniste » qui assimile les livres des juristes romains à des textes normatifs, pour en saisir en revanche les péripéties en tant que littérature. Mon souhait est de redonner corps à cette littérature, qui tend sinon à être réduite à son contenu de règlementation juridique.

- Vos recherches actuelles, notamment à la direction de l'ERC Redhis', réévalue la place des juristes dans la culture tardive. Comment étudier le droit romain tardif, notamment à

1 Rediscovering the hidden structure - A New Appreciation of Juristic Texts and Patterns of Thought in Late Antiquity. 
partir des sources papyrologiques, peut modifier notre perception de l'Antiquité tardive, dans toute sa complexité?

Le projet Redhis (Rediscovering the hidden structure) financé par l'Union européenne porte sur la présence dans l'Antiquité tardive ( $\mathrm{III}^{\mathrm{e}}-\mathrm{VI}^{\mathrm{e}}$ siècles) d’une " structure cachée ", qui, à mon sens, est constituée par le droit élaboré par les juristes romains. La question au cœur du projet est donc celle-ci : est-ce que cette structure a survécu à la fin de la production de nouveaux livres par les juristes, ce qui a eu lieu à peu près au milieu du III ${ }^{\mathrm{e}}$ siècle après Jésus-Christ? La réponse doit être cherchée dans les papyrus parce que, s’il est vrai que les juristes ont cessé d'écrire de nouveaux ouvrages avec très peu d'exception après la première moitié $d u{ } \mathrm{II}^{\mathrm{e}}$ siècle, il n'en est pas moins vrai que, entre Dioclétien et Justinien, les livres des juristes qui avaient été écrits auparavant ont continué à être copiés et lus, en Occident et en Orient. Ainsi le droit romain classique est demeuré le droit romain en vigueur jusqu'à la fin de l'Antiquité, cité dans les tribunaux, étudié dans les écoles. Le Digeste vu sous cet angle n'est pas une récupération hors du temps et même sans raison d'être, comme souvent on le considère, une sorte de rêve classiciste de Justinien au $\mathrm{vI}^{\mathrm{e}}$ siècle, son regard figé dans un passé révolu. Il n'est que l'aboutissement naturel de la familiarité que les Romains de toutes les époques ont eu avec cette littérature. Cette familiarité n'a jamais connu une crise fatale et une césure telles qu'on se les imagine, justement parce que cette littérature a continué à circuler. Notre projet, qui combine les savoirs des juristes et les savoirs de papyrologues et de paléographes, nous en révèle la richesse inattendue: nous avons identifié des textes de juristes romains dans une trentaine de papyrus jusqu'ici inédits. Cela implique un élargissement de la base textuelle qui n'est pas du tout négligeable, dans une discipline qui ne connaît pas souvent ce type d'enrichissement. En même temps, l'ensemble de ces données peut, je l'espère, amener à changer un peu de perspective. Il faut dès lors tenir compte de cette continuité qui a caractérisé la culture juridique, même pendant l'Antiquité tardive. La législation impériale, qui était très riche, s'insérait dans un cadre déjà bien rempli par les livres des juristes, qui, copiés, lus, glosés, constituaient cette « structure » maintenant un peu moins « cachée ».

\author{
Hélène Ménard \\ Maître de conférences \\ d'Histoire romaine à l'Université \\ Montpellier 3 - Paul Valéry \\ helene.menard@univ-montp3.fr
}

